\title{
Finite Toda lattice and classical moment problem
}

\author{
A. S. Mikhaylov ${ }^{1,2}$, V.S. Mikhaylov ${ }^{1}$ \\ ${ }^{1}$ Saint Petersburg Department of V. A. Steklov Institute of Mathematics of the Russian Academy of Sciences, \\ 7, Fontanka, 191023 Saint Petersburg, Russia \\ ${ }^{2}$ Saint Petersburg State University, Saint Petersburg State University, 7/9 Universitetskaya nab., \\ Saint Petersburg, 199034 Russia \\ mikhaylov@pdmi.ras.ru,vsmikhaylov@pdmi.ras.ru
}

DOI 10.17586/2220-8054-2020-11-1-25-29

We study the problem of computing the solution to finite Toda lattice. Specifically, we describe the evolution of moments of the spectral measure of a Jacobi matrix entering in the Lax pair.

Keywords: Toda lattice, moment problem, Jacobi matrices.

Received: 13 January 2020

Revised: 16 January 2020

\section{Introduction}

The semi-infinite or finite Toda lattice can be written in the following way:

$$
\left\{\begin{array}{l}
\dot{a}_{n}(t)=a_{n}(t)\left(b_{n+1}(t)-b_{n}(t)\right), \\
\dot{b}_{n}(t)=2\left(a_{n}^{2}(t)-a_{n-1}^{2}(t)\right), \quad t \geqslant 0, n=1,2, \ldots, N,
\end{array}\right.
$$

where $N \in \mathbb{N}$ or $N=\infty$, and one looks for a solution satisfying the initial conditions:

$$
a_{n}(0)=a_{n}^{0}, \quad b_{n}(0)=b_{n}^{0}, \quad n=1, \ldots, N,
$$

where $a_{n}^{0}, b_{n}^{0}$ are real and $a_{n}^{0}>0$. Toda lattices are used for modeling of nanosystems and macromolecules [1-3]. Methods of computing of functions $a_{n}(t), b_{n}(t)$ are subject of many investigations, see for example [4-6] and references therein. In the present paper, we restrict ourselves to the case of finite $N$, this situation was studied in [7].

We define two operators acting in $\mathbb{R}^{N}, f \in \mathbb{R}^{N}, f=\left(f_{1}, f_{2}, \ldots, f_{N}\right)$ by rules:

$$
\begin{aligned}
& H(t): f \mapsto\left\{\begin{array}{l}
a_{1}(t) f_{2}+b_{1}(t) f_{1}, \\
a_{n}(t) f_{n+1}+a_{n-1} f_{n-1}+b_{n}(t) f_{n}, \quad n=2, \ldots, N-1, \\
a_{N-1}(t) f_{N-1}+b_{N}(t) f_{N},
\end{array}\right. \\
& P(t): f \mapsto\left\{\begin{array}{l}
a_{1}(t) f_{2}, \\
a_{n}(t) f_{n+1}-a_{n-1}(t) f_{n-1}, \quad n=2, \ldots, N-1, \\
a_{N-1} f_{N-1} .
\end{array}\right.
\end{aligned}
$$

Note that the operator $H(t)$ is given by the Jacobi matrix (we keep the same notation for it):

$$
H(t)=\left(\begin{array}{ccccc}
b_{1}(t) & a_{1}(t) & 0 & 0 & 0 \\
a_{1}(t) & b_{2}(t) & a_{2}(t) & 0 & 0 \\
0 & a_{2}(t) & b_{3}(t) & a_{3}(t) & 0 \\
\cdot & \cdot & \cdot & \cdot & \cdot \\
0 & 0 & 0 & a_{N-1}(t) & b_{N}(t)
\end{array}\right)
$$

It is a well known fact $[5,6]$ that the system (1) is equivalent to the following operator equation:

$$
\frac{d H}{d t}=P H-H P \text {. }
$$

By $d \rho^{t}(\lambda)$, we denote the spectral measure of operator $H(t)$. Being a spectral measure of a bounded operator in $\mathbb{R}^{N}$, it has the form:

$$
d \rho^{t}(\lambda)=\sum_{k=1}^{N} \sigma_{k}^{2}(t) \delta\left(\lambda-\lambda_{k}(t)\right)
$$


where $\lambda_{k}(t), k=1, \ldots, N$ is a spectrum of $H(t)$. The moments of $d \rho^{t}(\lambda)$ are introduced by the rule:

$$
s_{k}(t)=\int_{-\infty}^{\infty} \lambda^{k} d \rho^{t}(\lambda), \quad k=0,1,2, \ldots
$$

It is well-known fact that the set of moments determines the semi-infinite Jacobi operator (but not necessarily in the unique way!), see [8,9] and $[10,11]$ for dynamic approach.

For infinite Toda lattices, people are interested in the evolution of scattering data for operator $H(t)[4,5,7]$. In the finite case in [7] the author studied the evolution of $\sigma_{k}(t)$ (it happens that $\lambda_{k}$ do not depend on $t$ ). In the present paper, we investigate the evolution of moments $s_{k}(t)$ under Toda flow. The authors are planning to use the obtained results for studying the semi-infinite Toda lattices, which will be the subject of forthcoming publications.

In the second section, we provide the necessary information on Toda lattices and adopt and rewrite some of results from [7] in a form, convenient for our purposes. In the last section, we remind the reader some basic facts on moment problem and derive the evolution equation for moments under the Toda flow.

\section{Finite Toda lattice, Moser formula.}

Here, we adapt some of results from [7] to the convenient forms for our use. For simplicity we usually omit the argument $t$.

Proposition 1. The eigenvalues of the matrix $H(t)$ do not depend on $t: \lambda_{j}(t)=\lambda_{j}(0)$.

This fact follows from the representation $\frac{d H}{d t}=i(H i P-(i P) H)=\{-i P, H\}$, and thus $H(t)=e^{P t} H(0) e^{-P t}$. Let $(\cdot, \cdot)$ denotes the scalar product in $\mathbb{R}^{N}$. The Weyl function $[12,13]$ is introduced by the rule:

$$
m(\lambda):=\left(R(\lambda) e_{1}, e_{1}\right)
$$

where:

with 1 being at $i$-th place.

$$
R(\lambda)=(H(t)-\lambda I)^{-1}, \quad e_{i}=(0, \ldots, 0,1,0, \ldots, 0),
$$

Proposition 2. The following relation holds:

$$
\frac{d}{d t} m(\lambda)=2 a_{1} R_{21}(\lambda)
$$

Proof. We can evaluate:

$$
\frac{d R}{d t}=-R \frac{d H}{d t} R=-R P H R+R H P R=-R P(I+\lambda R)+(I+\lambda R) P R=P R-R P .
$$

Then, using this relation, we have that:

$$
\frac{d}{d t} m(\lambda)=\left((P R-R P) e_{1}, e_{1}\right)=-2\left(R P e_{1}, e_{1}\right)=2 a_{1} R_{21} .
$$

We introduce the matrix:

$$
B_{N}=H-\lambda I=\left(\begin{array}{ccccc}
b_{1}-\lambda & a_{1} & 0 & 0 & 0 \\
a_{1} & b_{2}-\lambda & a_{2} & 0 & 0 \\
0 & a_{2} & b_{3}-\lambda & a_{3} & 0 \\
\cdot & \cdot & \cdot & \cdot & \cdot \\
0 & 0 & 0 & a_{N-1} & b_{N}-\lambda,
\end{array}\right)
$$

and minors $B_{k}, 1 \leqslant k<N$ of $B_{N}$, where $B_{k}$ are given by the intersection of $k$ rows $N-k+1, \ldots, N-1, N$ and $k$ columns $N-k+1, \ldots, N-1, N$. Denote $\Delta_{k}:=\operatorname{det} B_{k}$. Then, simple algebra shows that:

$$
\begin{array}{r}
m(\lambda)=R_{11}=\frac{\Delta_{N-1}}{\Delta_{N}}, \\
R_{21}(\lambda)=R_{12}(\lambda)=\left(R(\lambda) e_{1}, e_{2}\right)=-\frac{a_{1} \Delta_{N-2}}{\Delta_{N}} .
\end{array}
$$

Using these equalities we can rewrite (7) in the following form:

$$
\frac{d}{d t} m(\lambda)=2\left(1-\left(b_{1}-\lambda\right) m(\lambda)\right) .
$$


Representations of a Weyl function $[12,13]$ and a spectral measure (5) imply that:

$$
m(\lambda)=\int_{R} \frac{1}{\lambda-z} d \rho(z)=\sum_{k=1}^{N} \frac{\sigma_{k}^{2}(t)}{\lambda-\lambda_{k}} .
$$

Plugging the latter representation into (8) we have that:

$$
\sum_{k=1}^{N} \frac{2 \dot{\sigma}_{k} \sigma_{k}}{\lambda-\lambda_{k}}=2\left(1-\left(b_{1}-\lambda\right) \sum_{k=1}^{N} \frac{\sigma_{k}^{2}}{\lambda-\lambda_{k}}\right)
$$

where by dot we denote the differentiation with respect to $t$. Multiplying the last equality by $\left(\lambda-\lambda_{k}\right)$ and setting $\lambda=\lambda_{k}$, we come to the following system:

$$
\dot{\sigma}_{k}(t)=-\left(b_{1}-\lambda_{k}\right) \sigma_{k}(t), \quad k=1, \ldots, N .
$$

By $\|\cdot\|$ we denote the standard norm in $\mathbb{R}^{N}$.

Proposition 3. The coefficient $b_{1}$ admits the representation:

$$
b_{1}=\sum_{k=1}^{N} \lambda_{k} \sigma_{k}^{2}
$$

Proof. Denote by $C_{k}$ the eigenvectors of $H$ :

$$
H C^{k}=\lambda_{k} C^{k}, \quad C^{k}=\left(\begin{array}{c}
C_{1}^{k} \\
C_{2}^{k} \\
\ldots \\
C_{N}^{k}
\end{array}\right), \quad k=1, \ldots, N
$$

such that $\left\|C^{k}\right\|=1, k=1, \ldots, N$. Then, by the spectral theorem:

$$
C^{*} H C=\left(\begin{array}{ccccc}
\lambda_{1} & 0 & 0 & \ldots & 0 \\
0 & \lambda_{2} & 0 & \ldots & 0 \\
\ldots & \ldots & \ldots & \ldots & \ldots \\
0 & 0 & 0 & \ldots & \lambda_{N}
\end{array}\right), \quad \text { where } \quad C=\left(C^{1}\left|C^{2}\right| \ldots \mid C^{N}\right)
$$

i.e., the matrix $C$ is constructed from columns $C^{k}, k=1, \ldots, N$. Then:

$$
H=C\left(\begin{array}{ccccc}
\lambda_{1} & 0 & 0 & \ldots & 0 \\
0 & \lambda_{2} & 0 & \ldots & 0 \\
\ldots & \ldots & \ldots & \ldots & \ldots \\
0 & 0 & 0 & \ldots & \lambda_{N}
\end{array}\right) C^{*}
$$

from where and (3) we have that:

$$
b_{1}=H_{11}=\sum_{k=1}^{N} \lambda_{k}\left(C_{1}^{k}\right)^{2}=\sum_{k=1}^{N} \lambda_{k}\left(\sigma_{k}\right)^{2}
$$

The above proposition allows us to rewrite the system (9) in a more convenient form:

$$
\dot{\sigma}_{k}(t)=-\left(\sum_{j=1}^{N} \lambda_{j} \sigma_{j}^{2}(t)-\lambda_{k}\right) \sigma_{k}(t), \quad k=1, \ldots, N .
$$

Solution of (10) is given by the Moser formula:

$$
\sigma_{k}^{2}(t)=\frac{\sigma_{k}^{2}(0) e^{2 \lambda_{k} t}}{\sum_{j=1}^{N} \sigma_{j}^{2}(0) e^{2 \lambda_{j} t}}
$$




\section{Moment problem. Evolution of moments under the Toda flow.}

We denote by $C_{N}[X]$ the set of polynomials of order less than $N$. The set of moments $\left\{s_{k}\right\}_{k=0}^{2 N-2}$ determines on $C_{N}[X]$ the bilinear form by the rule: for $F, G \in C_{N}[X], F(\lambda)=\sum_{n=0}^{N-1} \alpha_{n} \lambda^{n}, G(\lambda)=\sum_{n=0}^{N-1} \beta_{n} \lambda^{n}$, one defines:

$$
\langle F, G\rangle=\sum_{n, m=0}^{N-1} s_{n+m} \alpha_{n} \beta_{m} .
$$

Thus this quadratic form is determined by the following Hankel matrix:

$$
S=\left(\begin{array}{ccccc}
s_{0} & s_{1} & s_{2} & \ldots & s_{N-1} \\
s_{1} & s_{2} & \ldots & \ldots & \ldots \\
s_{2} & \ldots & \ldots & \ldots & \ldots \\
\ldots & \ldots & \ldots & \ldots & s_{2 N-1} \\
s_{N-1} & \ldots & \ldots & s_{2 N-1} & s_{2 N-2}
\end{array}\right)
$$

In $[10,11]$ it is shown that $C_{N}[X]$ is in fact a de Branges space (we denote it here by $B^{N}(t)$ ), related to the dynamical system with discrete time associated with Jacobi matrix (3), see also [14,15]. The scalar product in $B^{N}(t)$ is given by $[F, G]_{B^{N}(t)}=\langle F, G\rangle$.

By $\|\cdot\|$ we denote the standard norm in $\mathbb{R}^{N}$. We introduce the vector:

$$
\widetilde{\sigma}(t)=\left(\begin{array}{c}
\widetilde{\sigma}_{1}(t) \\
\tilde{\sigma}_{2}(t) \\
\cdots \\
\tilde{\sigma}_{N}(t)
\end{array}\right)=\left(\begin{array}{c}
\sigma_{1}(0) e^{\lambda_{1} t} \\
\sigma_{2}(0) e^{\lambda_{2} t} \\
\ldots \\
\sigma_{N}(0) e^{\lambda_{N} t}
\end{array}\right)
$$

Then (11) and (14) implies that:

$$
\sigma_{k}(t)=\frac{\widetilde{\sigma}_{k}(t)}{\|\widetilde{\sigma}(t)\|}
$$

where

$$
\|\widetilde{\sigma}(t)\|=\sqrt{\sum_{j=1}^{N} \sigma_{j}^{2}(0) e^{2 \lambda_{j} t}}
$$

For $k=1, \ldots, N$ we have that:

$$
s_{k}(t)=\int_{\mathbb{R}} \lambda^{k} d \rho^{t}(\lambda)=\sum_{j=1}^{N} \lambda_{j}^{k} \sigma_{j}^{2}(t)=\sum_{j=1}^{N} \lambda_{j}^{k} \frac{\tilde{\sigma}_{j}^{2}(t)}{\|\widetilde{\sigma}(t)\|^{2}} .
$$

Then on introducing the notation

$$
\widetilde{s}_{k}(t)=s_{k}(t)\|\widetilde{\sigma}(t)\|^{2},
$$

and using (15) we see that

$$
\dot{\widetilde{s}}_{k}(t)=\sum_{j=1}^{N} \lambda_{j}^{k} 2 \dot{\widetilde{\sigma}}_{j}(t) \widetilde{\sigma}_{j}(t)=\sum_{j=1}^{N} \lambda_{j}^{k+1} 2 \widetilde{\sigma}_{j}^{2}(t)=2 \widetilde{s}_{k+1}(t) .
$$

We take $F, G \in C_{N}[X], F(\lambda)=\sum_{n=0}^{N-1} \alpha_{n} \lambda^{n}, G(\lambda)=\sum_{n=0}^{N-1} \beta_{n} \lambda^{n}$, then the scalar product in $B^{N}(t)$ has the form:

$$
[F, G]_{B^{N}(t)}=\sum_{n, m=0}^{N-1} s_{n+m}(t) \alpha_{n} \beta_{m} .
$$

We multiply both sides of the above equality by $\|\widetilde{\sigma}(t)\|^{2}$ and differentiate:

$$
\begin{array}{r}
\left([F, G]_{B^{N}(t)}\|\widetilde{\sigma}(t)\|^{2}\right)^{\prime}=\sum_{n, m=0}^{N-1}\left(\|\widetilde{\sigma}(t)\|^{2} s_{n+m}(t)\right)^{\prime} \alpha_{n} \beta_{m} \\
=\sum_{n, m=0}^{N-1}\left(\widetilde{s}_{n+m}(t)\right)^{\prime} \alpha_{n} \beta_{m}=\sum_{n, m=0}^{N-1} 2 \widetilde{s}_{n+m+1}(t) \alpha_{n} \beta_{m}=2\|\widetilde{\sigma}(t)\|^{2} \sum_{n, m=0}^{N-1} s_{n+m+1}(t) \alpha_{n} \beta_{m} .
\end{array}
$$


Differentiating the left hand side of the above equality, we have that:

$$
\left([F, G]_{B^{N}(t)}\|\widetilde{\sigma}(t)\|^{2}\right)^{\prime}=\left(\|\widetilde{\sigma}(t)\|^{2}\right)^{\prime} \sum_{n, m=0}^{N-1} s_{n+m}(t) \alpha_{n} \beta_{m}+\|\widetilde{\sigma}(t)\|^{2} \sum_{n, m=0}^{N-1} \dot{s}_{n+m}(t) \alpha_{n} \beta_{m} .
$$

On equating (18) and the right and side of (17), we come to the relation:

$$
\frac{\left(\|\widetilde{\sigma}(t)\|^{2}\right)^{\prime}}{\|\widetilde{\sigma}(t)\|^{2}} \sum_{n, m=0}^{N-1} s_{n+m}(t) \alpha_{n} \beta_{m}+\sum_{n, m=0}^{N-1} \dot{s}_{n+m}(t) \alpha_{n} \beta_{m}=2 \sum_{n, m=0}^{N-1} s_{n+m+1}(t) \alpha_{n} \beta_{m} .
$$

Due to the arbitrariness of $F, G$ the last equality implies that for moments $s_{k}$ the following system holds:

$$
\dot{s}_{k}(t)+\left(\ln \left\{\|\widetilde{\sigma}(t)\|^{2}\right\}\right)^{\prime} s_{k}(t)=2 s_{k+1}(t), \quad k=0, \ldots, 2 N-2 .
$$

Since we know that $s_{0}(t)=1$ for all $t$, then (19) allows us to determine $s_{1}(t), s_{2}(t) \ldots, s_{2 N-2}(t)$ recursively. Then, we use the fact that the set of moments $s_{k}(t), k=0, \ldots, 2 N-2$ determines $N \times N$ Jacobi matrix (3) and thus coefficients $a_{k}(t), b_{k}(t), a_{N}(t), k=1, \ldots, N-1$. Formulas for the reconstruction of entries of Jacobi matrix from moments are given in $[8,14,16]$

Analysis of the solution to (19) as well as an application of the results of the present paper and of $[11,16]$ to the case of semi-infinite Toda lattices will be the subject of forthcoming publications.

\section{Acknowledgements}

A. S. Mikhaylov and V.S. Mikhaylov were partially supported by RFBR 18-01-00269 and by the Ministry of Education and Science of Republic of Kazakhstan under grant AP05136197 and by Volkswagen Foundation project "From Modeling and Analysis to Approximation".

\section{References}

[1] Cho K. Optical Response of Nanostructures.: Microscopic Nonlocal Theory. Springer Science \& Business Media, Berlin, 2003.

[2] Muto V., Scott A.C., Christiansen P.L. A Toda lattice model for DNA: Thermally generated solitons. Physica D: Nonlinear Phenomena, 1990, 44(1-2), P. 75-91.

[3] Tsen K.-T. (Ed.) Non-Equilibrium Dynamics of Semiconductors and Nanostructures. CRC Press, 2018.

[4] Faddeev L.D., Takhtajan L.A. Hamiltonian Methods in the Theory of Solitons. Springer-Verlag Berlin Heidelberg, 2007.

[5] Teschl G. Jacobi operator and completely integrable nonlinear lattices. Providence: Amer. Math. Soc., 2001.

[6] Toda M. Theory of Nonlinear Lattices. Springer Series in Solid-State Sciences, 20 (2 ed.), Berlin: Springer, 1989.

[7] Moser J. Finitely many mass points on the line under the influence of an exponential potential - an integrable system. Dynamical Systems, Theory and Applications, Lecture Notes in Physics, 1975, 38, P. 67-497.

[8] Akhiezer N.I. The classical moment problem and some related questions in analysis. Edinburgh, Oliver and Boyd, 1965.

[9] Simon B. The classical moment problem as a self-adjoint finite difference operator. Advances in Math., 1998, 137, P. 82-203.

[10] Mikhaylov A.S., Mikhaylov V.S. On application of the Boundary control method to classical moment problems. Journal of Physics: Conference Series - IOPscience.

[11] Mikhaylov A.S., Mikhaylov V.S. Inverse problem for dynamical system associated with Jacobi matrices and classical moment problems. https://arxiv.org/abs/1907.11153

[12] Gesztesy F., Simon B. $m$-functions and inverse spectral analisys for finite and semi-infinite Jacobi matrices, J. d'Analyse Math., 1997, 73, P. 267-297.

[13] Mikhaylov A.S., Mikhaylov V.S., Simonov S.A. On the relationship between Weyl functions of Jacobi matrices and response vectors for special dynamical systems with discrete time. Mathematical Methods in the Applied Sciences, 2018, 41(16), P. 6401-6408.

[14] Mikhaylov A.S., Mikhaylov V.S. Inverse dynamic problems for canonical systems and de Branges spaces. Nanosystems: Physics, Chemistry, Mathematics, 2018, 9(2), P. 215-224.

[15] Mikhaylov A.S., Mikhaylov V.S. Boundary Control method and de Branges spaces. Schrödinger operator, Dirac system, discrete Schrödinger operator. Journal of Mathematical Analysis and Applications, 2018, 460(2), P. 927-953.

[16] Mikhaylov A.S., Mikhaylov V.S. Dynamic inverse problem for Jacobi matrices. Inverse Problems and Imaging, 2019 , 13(3), P. $431-447$. 